\title{
Labyrinthe
}

$6 \mid 2000$

Numéro 6

De l'inexistence de « l'art religieux » à l'avènement de l'archéologie de la religion

\section{Marie-Laure Portal}

\section{(2) OpenEdition \\ 12 Journals}

Édition électronique

URL : http://journals.openedition.org/labyrinthe/437

DOI : $10.4000 /$ labyrinthe.437

ISSN : 1950-6031

Éditeur

Hermann

Édition imprimée

Date de publication : 1 juin 2000

Pagination : 103-110

\section{Référence électronique}

Marie-Laure Portal, « De l'inexistence de « l'art religieux » à l'avènement de l'archéologie de la religion », Labyrinthe [En ligne], 6| 2000, mis en ligne le 18 mars 2005, consulté le 30 avril 2019. URL : http:// journals.openedition.org/labyrinthe/437 ; DOI : 10.4000/labyrinthe.437

Ce document a été généré automatiquement le 30 avril 2019

Propriété intellectuelle 


\title{
De l'inexistence de «l'art religieux » à l'avènement de l'archéologie de la religion
}

\author{
Marie-Laure Portal
}

1 Les ouvrages destinés aux dieux font partie de ceux qu'on a pris globalement le plus grand soin à confectionner, qu'on a en général construits avec les matériaux les plus solides et les plus beaux ${ }^{1}$, de sorte qu'ils se conservent plus fréquemment que l'équipement domestique par exemple, au point que l'art de l'Antiquité se réduit souvent pour le néophyte à une liste sans fin de sanctuaires. Cet état de fait relève non d'une organisation inhérente aux ouvrages en cause, mais des contraintes propres à notre condition d'observateur rétrospectif, limité à un seul temps, donc incapable de saisir (en dehors de sources testimoniales) la globalité des équipements produits en une situation historique donnée ${ }^{2}$.

2 On ne peut donc que constater la place à part que semble occuper le secteur religieux dans l'ensemble de la production artistique. Mais constater n'est pas entériner et l'apparente inertie de nos disciplines ne saurait être un obstacle à une remise en question quelle qu'elle soit. Le point de vue développé sur ce sujet découle logiquement de présupposés bâtis sur une refondation globale de l'archéologie donc débordant largement la question restrictive présentée ici, et sur les conséquences induites par ces conceptions. J'adopte pour répondre à la question de l'existence d'un « art religieux » deux points de vue successifs, qu'il ne faut jamais prendre l'un pour l'autre au risque d'une confusion intellectuelle, là où le but réside dans la dissociation analytique : le premier, d'ordre logique, celui de la science et le second, d'ordre sociologique, celui du métier. Cela m'amènera enfin à parler de l'archéologie de la religion.

3 Poser la question de l'existence d'un " art religieux » revient à se demander si on est fondé à isoler scientifiquement un secteur à part au sein de l'objet de l'archéologie. La réponse à ce problème dépend de la définition de celui-ci. 

(au sens latin d'ars), qui apparaît comme la plus justifiée et la plus cohérente l'artificialité constitue le critère définitoire de l'archéologie quelles que soient les façons dont on accède à l'observation (par la fouille, par tout autre examen direct, ou bien par les sources testimoniales). Analytiquement, le technique n'est pas le social : il s'agit de deux capacités rationnelles autonomes même si elles interagissent l'une sur l'autre.

7 L'impropriété constitutive du langage est un obstacle à toute analyse puisque le même mot qualifie plusieurs objets (polysémie) et qu'un même objet se nomme différemment (synonymie). Cette difficulté est exemplairement illustrée par le terme " art ». En effet, celui-ci s'emploie dans plusieurs acceptions : il désigne toute production de l'activité outillée ou un savoir-faire particulier (« les arts et métiers ») ou encore le jugement valorisé qu'on porte sur un objet (« la littérature est de l'Art »; on parle des « Beaux-Arts »). La définition de l'archéologie par l'étude de l'art comme capacité rationnelle d'accès à la technique implique qu'on prend en considération indifféremment les choses valorisées et les objets jugés « ignobles » (dans l'acception étymologique du mot). On ne saurait donc établir scientifiquement une classification artistique sur ce critère.

8 La notion d'Art fait très souvent de l'étude des ouvrages une histoire des artistes : on se sert ainsi de leur vie pour expliquer leur production artistique. Cet engouement explique la recherche effrénée de l'attribution des œuvres. Mais cela revient à nier l'effet propre de la technique qui ne serait qu'un simple reflet des pensées de l'artiste dans sa production. Or ce qui se fabrique échappe bien souvent à la conscience de ce dernier : l'art en tant qu'il est analyse structure à sa façon pensée et société, donc les modifie. La vie des artistes relève de la seule histoire et c'est à l'archéologie que revient l'analyse des ouvrages : cette dernière science doit en conséquence n'étudier que les œuvres en faisant abstraction de leur auteur.

et la religion ressortissent à deux capacités autonomes : la technique et la sociologie (entendue comme analyse de l'appartenance à une communauté, quelle que soit son étendue). C'est de ces deux points de vue qu'il faut se placer.

Du point de vue de l'art, si on prend en compte tout l'équipement, indépendamment de la valeur artistique qu'on lui accorde, il est clair qu'on utilise les mêmes techniques pour faire un tableau figurant un saint ou une scène de genre ; les mêmes maçons construisent le bistrot aussi bien que l'église. Les thèmes religieux des images sont uniquement une catégorie parmi une infinité d'autres référents imagiers. Mis à part les images, l'équipement employé dans le cadre religieux se distingue uniquement par la destination qu'on lui donne, mais celle-ci ne saurait être un critère définitoire puisqu'elle n'est pas incluse dans la fabrication : les ciboires et autres patènes ne se différencient pas techniquement de la riche vaisselle utilisée au Moyen Âge ; ce qui varie, c'est la conjoncture puisque, dans le premier cas, les objets servent à la communion des fidèles tandis que, dans le second, ils sont employés dans le cadre profane. Les contraintes qui pèsent sur la production à destination religieuse par le biais de l'orthodoxie sont certes 
particulièrement lourdes ; si elles apparaissent tellement prégnantes, c'est en raison du caractère édicté de ce type de réglementation. Mais l'existence de normes implicites ne saurait être mise en doute quel que soit le contexte de la production artistique : tout ne se montre pas dans les tableaux, même si aucun code écrit ne l'explicite ouvertement.

Du point de vue sociologique, la religion est un phénomène social comme un autre : il s'agit de se réunir entre fidèles d'une même communauté. Cela ressortit au même processus qui fait que des individus appartiennent à un groupe tout en étant exclus d'un autre selon le critère considéré. Par exemple, catholiques et protestants forment une communauté chrétienne : sous cet aspect, ils sont identiques socialement. Mais les critères d'appartenance sociale sont infiniment variables de sorte que, sur tel point deux individus relèvent du même groupe social et sur tel autre de deux groupes différents ; ainsi, unitaires dans la communauté chrétienne, les individus de ce groupe relèveront d'autres communautés, par exemple sous le critère professionnel.

De ce fait, parce qu'il n'y a autonomie ni par la technique mise en jeu, ni par le type de mécanisme sociologique en cause, parler d'un " art religieux " ne repose sur aucun fondement scientifique. Cela apparaît symptomatiquement dans la multiplication de pseudo-critères définitoires : ainsi de l'exclusion thématique des scènes de genre, l'emploi d'un savoir-faire particulier (comme la fresque) ou encore les mœurs irréprochables de l'artiste ; ce flou illustre l'absence d'un critère définitoire scientifiquement établi.

Si science archéologique il y a, il n'est dans les faits que des archéologues; il ne faut donc pas attribuer à l'une ce qui ressortit aux autres. Scientifiquement, en effet, tous les objets d'étude se valent sans impliquer aucune hiérarchie des uns et des autres, mais professionnellement il est évident que chacun ne peut s'occuper de tout, ni s'intéresser à tout, ni savoir pratiquer toutes les démarches archéologiques. L'arbitraire des choix de chacun est une affaire qui n'a rien à voir avec la science, de sorte que, si scientifiquement " l'art religieux " n'existe pas, il est loisible à chacun de préférer étudier ce type d'équipement : du point de vue de la science, il n'y a pas « d'art religieux », mais, du point de vue du métier d'archéologue, il peut y avoir une archéologie de la religion.

Dès lors, il faut s'interroger sur les extensions de ce champ, car, si chacun peut n'étudier qu'une partie de la question, le chercheur n'est pas libre des connexions inhérentes à son objet d'étude.

Dans l'archéologie, le champ de la religion comprend l'équipement d'un phénomène qui, avant d'être artificialisé, est principalement religieux ${ }^{3}$. L'adhésion religieuse passe par deux voies : celle de la croyance qui, visant à démontrer apologétiquement l'existence du dieu, donne lieu à un enseignement d'instruction (de Vérités à croire) et d'édification (de biens moraux à désirer), et celle du culte qui, établissant mystiquement la présence du dieu, donne lieu à une fréquentation. L'équipement de la religion s'ordonne donc suivant ces deux fonctions.

D'une part, l'équipement de la croyance : il outille le caractère proclamé ou exposé de l'enseignement. Cela est illustré, dans le premier cas, par la salle d'initiation que constitue le Télestérion d'Éleusis chez les Grecs ou par l'équipement de l'homélie (chaire à prêcher, etc.) et de la lecture dans le cas d'une religion révélée comme le catholicisme ; dans le second cas, il s'agit d'exploiter les inscriptions pour la catéchèse, comme celles inscrites sur le fronton du temple d'Apollon à Delphes, ou bien les images : elles sont moralisatrices (par exemple les trois chemins d'éternité) ou didactiques, sur la nature et 
l'histoire du dieu (métopes du temple de Zeus à Olympie, chemin de croix de Pontchâteau ${ }^{4}$, etc.).

17 D'autre part, les ouvrages du culte : ils équipent éventuellement la présence divine et la rencontre avec le dieu. Celui-ci peut être rendu magiquement présent auprès de son fidèle au moyen d'une image, d'une relique (ainsi de l'autel de cornes d'Apollon à Délos) ou, chez les catholiques, grâce à l'eucharistie. La fréquentation du dieu ne suppose pas un équipement particulier, mais ce dernier abonde bien souvent : ce domaine est bien plus vaste qu'on ne le pense spontanément et comprend aussi bien les vêtements liturgiques que ce qui abrite la rencontre (chapelle, église entière, etc.), équipe le sacrifice (réel dans l'Antiquité ou mystique chez les catholiques) ou l'échange (ex-voto, inscriptions des oraisons, etc.).

Les intérêts particuliers des uns et des autres accommodent les objectifs scientifiques en priorités de recherche. Dans tous ses champs d'étude, l'archéologie cherche à faire apercevoir les distorsions que l'art fait subir aux hiérarchies sociales instituées (ainsi dans mon article sur « L'apport de l'archéologie à l'étude du culte marial parisien » avec le cas de la Vierge et de la pyramide sainte) et aux conceptions : par exemple, le tableau Les Ruines (v. 1885) de James Tissot figurant le Christ et deux communards dans les ruines de la Cour des Comptes (après son incendie en 1871) modifie l'idée que nous nous faisons de Lui du fait de cette récupération politique suggérant Sa sympathie pour les communards. Un des plus grands bénéfices d'une archéologie traitant de la religion consiste à mettre en lumière les transgressions que s'autorise l'équipement par rapport aux règles édictées par l'orthodoxie.

19 Au terme de cet examen, redisons que l'emploi indu de l'expression " art religieux » provient de l'entremêlement de questions qu'il faut nécessairement dissocier sous peine de prendre pour ressortissant à l'une ce qui relève de l'autre : il n'y a aucun fondement scientifique pour reconnaître à " l'art religieux " une autonomie dans l'objet de l'archéologie, bien que chacun puisse à son gré préférer s'occuper de l'équipement de la religion. Cessons donc d'attribuer à la science ce qui n'est qu'une question de convenance personnelle.

20 Outre le raisonnement de ce problème restreint, l'exercice est intéressant pour l'explicitation des présupposés qu'il met en œuvre. C'est là, me semble-t-il, le véritable enjeu de ce type de débat. En effet, la prise de position en faveur d'un « art religieux » ou de son inanité repose sur des conceptions précises de notre discipline. Pour ma part, je défends la nécessité de redéfinir l'archéologie ; cela apporte plusieurs avantages illustrés par la question qui est débattue ici.

21 Tout d'abord, la refondation de l'archéologie selon l'autonomie d'une capacité technique analogue à celle du langage, de la société ou du droit met en évidence la dissociation de questions habituellement traitées conjointement. En premier lieu, l'art présente une organisation rationnelle propre, de sorte qu'un ouvrage ne se contente pas de traduire passivement la volonté d'un artiste : par le biais de la technicisation, une partie de ce qui est fabriqué échappe à la conscience de son créateur ; on ne saurait considérer une œuvre uniquement dans son rapport à son fabricant (peu importe ici qu'il soit qualifié socialement d'artiste ou d'artisan). Et de même du destinataire de l'ouvrage : que le fidèle établisse une dévotion vis-à-vis d'un certain tableau ou qu'il reste froid devant lui ne change rien à ce qui a été fabriqué. Ces questions du fabricant et du destinataire se posent évidemment, mais ressortissent avant tout à l'histoire et non à l'archéologie ; on ne saurait raisonner un objet d'étude de l'archéologie en fonction d'un autre, ressortissant à 
l'histoire. En second lieu, la définition de l'archéologie par l'ars n'implique en soi aucunement une quelconque valorisation de la production artistique : ce sont deux problèmes distincts. En éliminant de la définition de l'archéologie la question de l'Art, on y gagne de ne plus plaquer un regard rétrospectif, critique et incessamment fluctuant sur les ouvrages d'après le critère de leur importance historique.

À distinguer analytiquement l'organisation inhérente à un ouvrage, on s'affranchit également des manifestations historiquement particulières d'un phénomène, comme on a $\mathrm{pu}$ le voir ici avec ce que serait une archéologie de la religion. De cette façon, il devient possible de prendre du recul par rapport à un cas particulier pour apercevoir des mécanismes génériques qui se retrouvent dans des conditions historiques infiniment variées ; en posant la question de l'archéologie de la religion comme cela a été fait ici, il devient possible de rapprocher les cas complexes de l'équipement des religions grecque ou romaine et du catholicisme par exemple : en soi, ces phénomènes sont irréductibles l'un à l'autre, mais on les rapporte à des processus plus vastes de sorte qu'on compare analytiquement des ouvrages pourtant configurativement disparates.

\section{NOTES}

1.Par exemple les comptes du sanctuaire de Délos mettent en évidence la riche dotation du dieu, propriétaire de nombreuses fermes mises en location, destinataire de somptueux cadeaux, etc.

2.Je me réfère dans tout l'article aux positions défendues dans RAMAGE (Revue d'Archéologie moderne et d'Archéologie générale), fascicules 1 à 13, publiés aux P.U.P.S. (Presses Universitaires de Paris-Sorbonne), reprises systématiquement dans MAGE ( Mémoires d'Archéologie générale) publié aux P.U.P.S. (Paris, 1996) par Ph. Bruneau et P.-Y. Balut.

3.Je me réfère aux cours dispensés en 1997-98 par Ph. Bruneau à Paris IV-Sorbonne. Dans le cas particulier de la religion catholique, on peut notamment se reporter aux articles de Ph. Bruneau : « Linéaments d'une archéologie du catholicisme », RAMAGE, 4 (1986), p. 127-149 et « Qu'est-ce qu'une église ? », RAMAGE, 9 (1991), p. 49-84.

4.Sur cet exemple précis, voir Ph. Bruneau, «Le calvaire de Pontchâteau », RAMAGE, 2 (1983), p. 11-41. 


\section{AUTEUR}

\section{MARIE-LAURE PORTAL}

Étudiante de troisième cycle à Paris IV, auteur dans le numéro 4 de Labyrinthe de l'article « Apport de l'archéologie à l'étude du culte marial parisien pour l'époque contemporaine $\gg$. 